\title{
Gestão Estratégica de Quadros: as «Práticas de Trabalho de Elevado Desempenho»
}

\author{
João Pina Cordeiro \\ joao.cordeiro@esce.ips.pt \\ Escola Superior de Ciências Empresariais \\ Instituto Politécnico de Setúbal
}

\begin{abstract}
Resumo
Esta comunicação reflecte sobre a gestão estratégica de quadros. O objectivo geral do estudo é analisar as inter- relações entre as «práticas de trabalho de elevado desempenho»(Becker, Huselid, Ulrich, 2001; Pil e MacDuffie, 1996) e as estratégias empresariais. O objecto central do estudo são as empresas do sector de componentes para automóvel, nomeadamente os seus quadros superiores (QS). A técnica de investigação utilizada foi o inquérito por questionário, ao qual responderam 72 empresas do sector de componentes para automóvel. Os resultados indicam que a diferente conjugação das dimensões definidoras da gestão estratégica de quadros (participação/acesso à informação, segurança no emprego, formação profissional, gestão de carreiras e gestão das remunerações), está na base de dois modelos contrastantes, a saber: um que inclui as empresas com estratégia assente na "inovação" e na "qualidade" e outro que inclui as empresas com estratégia assente nos "baixos custos". Concluímos que as empresas, ao possuírem diferentes estratégias de negócio, possuem também diferentes formas de preconizar essas mesmas estratégias, as quais sustentam e são sustentadas por diferentes práticas de trabalho de elevado desempenho, num processo de retroalimentação mútua. Este estudo contribui para uma melhor compreensão da teoria envolvendo as estratégias empresariais e a gestão de quadros, permitindo aprofundar a compreensão sobre as práticas de trabalho de elevado desempenho e aperfeiçoar o processo de planeamento dos sistemas de gestão de quadros nas empresas.
\end{abstract}

Palavras chave: Gestão Estratégica de Quadros, Práticas de Trabalho de Elevado Desempenho, Sector de Componentes para Automóvel

\section{Introdução}

Uma das fontes de vantagem competitiva mais importante refere-se à capacidade e/ou à habilidade da organização para gerir os seus quadros. É um facto que as empresas têm alterado significativamente o modo como gerem os seus quadros, preconizando práticas de gestão estratégica centradas na valorização do factor humano - e.g. gestão estratégica dos recursos humanos (Bilhim, 2004).

A definição do modelo de gestão pressupõe, assim, que a empresa pense e decida, em cada momento do seu ciclo de vida, sobre as estratégias (onde se incluem os objectivos das empresas) que lhe permita alcançar os objectivos definidos, bem como as práticas de gestão dos recursos humanos que lhe estão associadas, num processo de retroalimentação mútua. Dado que a qualificação dos recursos humanos de uma entidade é um dos maiores garantes de sucesso do 
negócio, é necessário manter presente que a gestão dos recursos humanos não se esgota na contratação. É, igualmente, importante manter os trabalhadores motivados no desempenho das funções através de práticas de acesso à informação, de gestão da formação, de gestão da remuneração e de gestão das carreiras qualificantes.

Esta comunicação tem por base uma investigação inserida no Doutoramento em Sociologia sob o tema "Estratégias empresariais e gestão de recursos humanos" (Cordeiro, 2008). Apresentamos aqui alguns dados sobre a relação entre as «práticas de trabalho de elevado desempenho» (PTED) e as «estratégias empresariais» em empresas do sector de componentes para automóvel. Estruturalmente, começamos por traçar o quadro teórico que sustenta a análise, seguindo-se uma referência à metodologia utilizada e à apresentação dos resultados. Concluímos com uma síntese dos resultados mais relevantes sobre as relações encontradas.

\section{Secção 1 - Enquadramento Teórico}

\section{A gestão estratégica de quadros: as «práticas de trabalho de elevado desempenho»}

Foi a partir de meados da década de 80 que surgiu uma maior preocupação com o nível estratégico da gestão das pessoas nas empresas (Huselid et al., 1997; Huang, 2002), assistindo-se à transformação do papel dos recursos humanos $(\mathrm{RH})$ das organizações, passando de uma actuação operacional para uma actuação estratégica (Bilhim, 2004).

A gestão estratégica de RHs pressupõe a inter-relação entre a gestão das pessoas e a estratégia organizacional. Contempla, assim, duas dimensões: a dimensão vertical, onde a estratégia de gestão de RH deverá ser coerente e devidamente integrada com a estratégia de negócio da empresa, e a dimensão horizontal, que enfatiza a coerência e integração das diferentes políticas funcionais da gestão de RH entre si, que não podem contradizer-se nos fins que visam nem nos efeitos que produzem (Wright e McMahan, 1992; Becker e Huselid, 1999).

Uma das características da nova política de gestão estratégica refere-se ao papel fulcral que os profissionais mais qualificados (quadros e não só) assumem enquanto parte integrante e integradora da actividade das empresas (Torrington, 2002). Neste sentido, a estrutura organizacional deve procurar delinear e implementar um sistema de gestão de quadros que lhe 
permita adequar a componente humana aos objectivos estratégicos da organização, para o que é necessário existir uma boa relação entre a estratégia de recursos humanos e a estratégia global da empresa" (Pernin, 1985:74).

A consideração da gestão de quadros como um conjunto de práticas é uma das abordagens conceptuais que decorre de duas razões principais. Em primeiro lugar, da necessidade de conceptualizar um conjunto muito diversificado de práticas e dos seus respectivos resultados, obtidos através das pessoas, que se constituem como factor de vantagem competitiva. Em segundo lugar, da necessidade de controlar os seus efeitos na organização, não podendo dissociar deste processo factores como a cultura e a estratégia da organização.

As práticas, a este nível, correspondem a um conjunto de ferramentas e de instrumentos que fazem parte de um sistema de gestão das pessoas que procure analisar e potenciar as habilidades dos trabalhadores, os seus conhecimentos e a sua experiência, e remetem para o que alguns autores denominam por Práticas de Trabalho de Elevado Desempenho (Becker, Huselid, Ulrich, 2001; Ciavarella, 2003; Pil e MacDuffie, 1996).

A ideia central das «PTED» consiste em fazer chegar até aos diferentes profissionais da organização, informação, conhecimento, recompensas e poder. Neste sentido, a implementação destas práticas depende das competências dos profissionais, dessas competências serem nucleares (core) para a estratégia da organização e dos profissionais terem motivação e oportunidades para as utilizar (Delery e Shaw, 2001).

Não havendo consenso quanto aos elementos caracterizadores das PTED é possível, contudo, agrupá-las em três grandes dimensões: a motivacional (compensação e gestão de carreiras); a participação (equipas autónomas, mecanismos de participação, job design) e o desenvolvimento (formação, avaliação de desempenho centrada no desempenho), todas com o objectivo claro de promover a inovação e a motivação do trabalhador, conduzindo a maior satisfação, comprometimento e a melhores resultados em termos de performance organizacional.

\section{Tipologias de estratégia}

De entre os muitos significados do conceito de estratégia, um dos mais utilizados é o de Thompson e Strickland (2000). Para eles, estratégia é um “conjunto de mudanças competitivas e 
abordagens comerciais que os gestores executam para atingir o melhor desempenho da empresa (...) para reforçar a posição da organização no mercado, promover a satisfação dos clientes e atingir os objectivos de desempenho" (Thompson e Strickland, 2000:10). Estratégia descreve as características fundamentais que as organizações definem, através das suas competências, recursos e oportunidades, que lhes permitem traçar metas e alcançar os seus objectivos.

No que se refere às tipologias de análise, saliente-se a teoria desenvolvida por Schuller e Jackson (1987). Partindo das premissas subjacentes ao trabalho de Porter $(1980,1985)$ sobre a estratégia competitiva, Schuller e Jackson (1987) desenvolvem três arquétipos da estratégia competitiva, estabelecendo relações entre essas estratégias e as práticas de gestão de recursos humanos, são elas: inovação, qualidade e redução de custos.

A estratégia de inovação é utilizada para desenvolver produtos e/ou serviços diferentes dos seus concorrentes. O seu foco principal está na oferta de algo novo e diferente, pelo que é fundamental que crie condições para favorecer a inovação. A estratégia de qualidade, que consiste em "fazer bem sempre", é utilizada para a melhoria de produtos e/ou serviços e de processos face aos seus concorrentes. O foco principal nesta estratégia está no incremento da qualidade, o que pressupõe que os trabalhadores estejam "engajados" com a qualidade e com a melhoria contínua. Na estratégia de redução dos custos as empresas obtêm vantagens competitivas por produzirem ao mais baixo custo, permitindo retornos acima da média. O baixo custo é fundamental, apesar da qualidade, assistência e outras áreas não poderem ser descuradas (Schuler e Jackson, 1987).

\section{Secção 2 - Metodologia}

Para este estudo recorreu-se a um inquérito por questionário, aplicado às empresas do sector de componentes para automóvel, composto por uma extensa bateria de indicadores, procurando-se inventariar a condição empresarial daquelas empresas e caracterizar as dimensões «estratégias empresariais» (estratégia principal) e «PTED» dos quadros superiores (QS).

No que se refere às variáveis utilizadas para concretizar a dimensão «estratégias empresariais», tendo por base a revisão da literatura, formulou-se a seguinte questão: Qual a estratégia principal da empresa para se adaptar ao mercado? Identificaram-se cinco opções de resposta: 
estratégia centrada no baixo custo; estratégia centrada na melhoria da qualidade; estratégia centrada na inovação; estratégia de foco; outra estratégia? Os tipos de estratégia considerados no inquérito por questionário remetem para os desenvolvimentos da teoria das vantagens competitivas de Porter (Porter, 1980, 1985) feitos por Schuler e Jackson (1987). No que se refere à identificação das estratégias empresariais, utilizámos ainda as seguintes variáveis: vantagens concorrenciais; posicionamento das empresas face aos produtos e aos processos; equipamentos/tecnologias; caracterização das empresas quanto à sua estrutura (Morais, 1998).

No que se refere às questões relacionadas com as «PTED» analisámos o processo na sua globalidade. Para o concretizar procurou-se analisar o processo de participação/acesso à informação, a segurança no emprego, a formação profissional, a gestão das carreiras e a gestão das remunerações.

Quanto à definição da amostra, não se pretende que ela seja representativa, mas que se adeqúe aos objectivos definidos. O que se verifica, pois cobre 72 empresas do sector, dispersas pelo País e de várias dimensões. De um total de cerca de 170 empresas do sector obteve-se uma taxa de resposta ao inquérito na ordem dos $42 \%$.

Para a análise dos dados do inquérito por questionário utilizou-se o programa estatístico para as ciências sociais - SPSS (Maroco, 2007).

\section{Secção 3 - Desenvolvimento}

A análise que se segue vai no sentido de identificar e caracterizar as «PTED», procurando verificar como se relacionam com as «estratégias empresariais».

\section{Participação/acesso à informação}

$\mathrm{Na}$ grande maioria das empresas existem reuniões periódicas de definição de estratégias/objectivos e de apresentação de resultados, sendo as funções de direcção (90\% e $91 \%)$ e de enquadramento superior $(71 \%$ e $82 \%)$ as que preferencialmente participam nesse processo. As funções de enquadramento participam, também, através de reuniões gerais de 
quadros, na proposta de métodos de organização de trabalho (85\%) e nas actividades/projectos relacionados com o funcionamento global da organização (74\%).

No que se refere ao papel dos QS na definição e na implementação das estratégias empresariais, as empresas possuem, maioritariamente, uma cultura de participação. Considerados agentes estratégicos de desenvolvimento da empresa (93\%), os QS participam na implementação das estratégias $(96 \%)$, e na sua definição $(71 \%)$.

Considerando a análise por estratégia empresarial, não encontramos diferenças no que se refere à participação dos QS na formulação da estratégia de negócio da empresa. Já no que se refere à participação na implementação da estratégia de negócio da empresa, os resultados indicam que são as empresas que assentam a sua estratégia na qualidade (3.46) e na inovação (3.56) as que tendem a valorizar mais o contributo dos QS, em contraste com as empresas que possuem estratégia de baixo custo (2.64), as quais se situam abaixo da média do grupo.

O teste de Kruskal Wallis revelou existirem diferenças estatisticamente significativas entre as empresas por tipo de estratégia $\left(X^{2}=13.887,2 \mathrm{gl}, \mathrm{p}<0.1\right)$. O contributo dos QS na implementação da estratégia varia nas empresas do sector automóvel e depende da estratégia empresarial. Nas empresas que adoptam predominantemente uma estratégia de qualidade ou de inovação reportam um contributo claramente maior dos QS na prossecução da estratégia a empresa.

\section{Segurança no emprego: práticas de renovação e tipo de contrato}

A grande maioria das empresas procedeu à contratação de QS nos últimos 3 anos (75\%), tendência que aumenta quando se caminha para empresas com estratégia principal de qualidade (76,9\%) e de inovação (87,5\%). Quando consideradas apenas as empresas com estratégia de baixo custo, e apesar da maioria revelar ter contratado QS nos últimos 3 anos $(58,8 \%)$, constatase que uma significativa percentagem não o fez.

Procurando reflectir sobre a questão da segurança no emprego, analisamos o "tipo de contrato de trabalho privilegiado no recrutamento de QS". Relativamente a esta variável, as empresas revelaram diferenças significativas consoante o tipo de estratégia adoptada (Tabela 1).

Enquanto as empresas de baixo custo tendem a privilegiar o contrato a termo para os QS $(62,5 \%)$, as empresas assentes em estratégias de qualidade e de inovação tendem a privilegiar a 
contratação sem termo de QS (51,3\% e 62,5\% respectivamente). A associação entre o tipo de contrato no recrutamento e o tipo de estratégia empresarial é estatisticamente significativa (Teste $V$ de Cramer $=.485, \mathrm{p}=.000$ ), ou seja, o tipo de contrato de trabalho privilegiado no recrutamento de QS varia nas empresas do sector e depende da estratégia.

Tabela 1 - Contrato de trabalho no recrutamento por estratégia empresarial

\begin{tabular}{llccccc}
\hline & \multicolumn{5}{c}{ Estratégia Empresarial } \\
\hline \multirow{3}{*}{$\begin{array}{l}\text { Contrato de trabalho privilegiado } \\
\text { no recrutamento de QS }\end{array}$} & Contrato a termo & Baixo Custo & Qualidade & Inovação & Total \\
\cline { 2 - 5 } & Contrato sem termo & $62,5 \%$ & $2,6 \%$ & $6,3 \%$ & $16,9 \%$ \\
& Período experimental para efectivo & $6,3 \%$ & $51,3 \%$ & $62,5 \%$ & $43,7 \%$ \\
& & \multirow{2}{*}{ Total } & $100,3 \%$ & $46,2 \%$ & $31,3 \%$ & $39,4 \%$ \\
& & & $100,0 \%$ & $100,0 \%$ & $100,0 \%$ \\
\hline
\end{tabular}

As empresas revelaram alguma homogeneidade quanto ao tipo de contrato dos QS (maioria opta por contrato sem termo). Todavia, existem algumas diferenças consoante o tipo de estratégia, pois, apesar das empresas de baixo custo terem maioritariamente QS com contrato sem termo, possuem também uma percentagem significativa com contrato a termo. Já as empresas com estratégias de qualidade e de inovação possuem maioritariamente QS com contratos sem termo

O teste de comparação de medianas de Kruskal Wallis revela diferenças estatisticamente significativas para os contratos sem termo e os contratos a termo entre as três estratégias não existindo, porém, diferenças significativas para o caso do recurso à prestação de serviços em QS (contrato sem termo - Kruskal-Wallis, $X^{2}=21.586,2 \mathrm{gl}$, p.000; contrato a termo - Kruskal-Wallis, $X^{2}=22.097,2 \mathrm{gl}$, p.000; prestação de serviços - Kruskal-Wallis, $X^{2}=.577,2 \mathrm{gl}, \mathrm{p} .749$ ).

A análise comparada da ordenação das medianas revela que as diferenças se devem aos valores apresentados pelas empresas com estratégia de baixo custo, que recorrem com maior intensidade a contratos a termo (com evidente redução no acesso à efectividade) e, consequentemente, maior. precariedade laboral.

\section{Segurança no emprego: práticas de saída}

Directamente relacionado com a questão da segurança no emprego analisou-se a saída de QS nos últimos 5 anos. Os resultados da tabela 2 indicam, claramente, que na esmagadora maioria das empresas não houve saída de qualquer QS nos últimos 5 anos $(78,2 \%)$. 
Tabela 2 - Saída de QS (últimos 5 anos) por estratégia empresarial

\begin{tabular}{lrrrrrr}
\hline & \multicolumn{5}{c}{ Estratégia Empresarial } \\
\hline \multirow{3}{*}{ Saída de QS nos últimos 5 anos } & & Baixo Custo & Qualidade & Inovação & \multirow{2}{*}{ Total } \\
\cline { 3 - 5 } & não & $58,8 \%$ & $82,1 \%$ & $81,3 \%$ & $78,2 \%$ \\
& sim & $41,2 \%$ & $17,9 \%$ & $18,7 \%$ & $22,8 \%$ \\
& Total & $\mathbf{1 0 0 , 0 \%}$ & $100,0 \%$ & $100,0 \%$ & $100,0 \%$ \\
\hline
\end{tabular}

A associação entre "saída de QS" e "tipo de estratégia" não é estatisticamente significativa. A variável "saída de QS" não depende da estratégia empresarial (Teste V de Cramer =.385, p>.05).

\section{Formação profissional}

\section{Realização de formação profissional}

As «PTED» são caracterizadas por oportunidades de formação e desenvolvimento. Neste sentido, analisou-se a realização de acções de formação profissional para os QS nos últimos 3 anos, diferenciando estes quadros por perfis (QS com perfil técnico - QSPT; e liderança QSPL). Os resultados da tabela 3 mostram que a grande maioria das empresas promoveu formação profissional nos últimos 3 anos para os QS, não evidenciando grandes diferenças de acordo com os perfis considerados (QSPT - 90,1\%; QSPL - 88,7\%). Todavia, as diferenças são evidentes se compararmos a realização de formação profissional por estratégia empresarial. A promoção de formação profissional é muito maior em empresas que com estratégia de qualidade (100\% QSPT; 97,4\% QSPL) e inovação (100\% ambos os perfis), do que nas empresas com estratégia de baixo custo (58,8\% ambos os perfis) (Tabela 3$)$.

Tabela 3 - Realização de formação profissional por estratégia empresarial

\begin{tabular}{lccccc}
\hline & \multicolumn{5}{c}{ Estratégia Empresarial } \\
\hline \multirow{2}{*}{ FP_ QSPT } & não & Baixo Custo & Qualidade & Inovação & Total \\
\cline { 3 - 5 } & sim & $51,2 \%$ & $0,0 \%$ & $0,0 \%$ & $9,9 \%$ \\
& Total & $100,0 \%$ & $100,0 \%$ & $100,0 \%$ & $90,1 \%$ \\
\cline { 2 - 5 } FP_ QSPL & não & $41,2 \%$ & $100,0 \%$ & $100,0 \%$ & $100,0 \%$ \\
& sim & $58,8 \%$ & $97,6 \%$ & $0,0 \%$ & $11,3 \%$ \\
& Total & $100,0 \%$ & $100,0 \%$ & $100,0 \%$ & $100,0 \%$ \\
\hline
\end{tabular}


Os resultados da associação $V$ de Cramer mostram que a realização de formação para os QS diverge entre as empresas com estratégia de baixo custo e as de estratégia de qualidade ou inovação. Existe uma associação estatisticamente significativa entre a estratégia e a realização de formação profissional (V de Cramer =.589, p.000 - QSPT; e V de Cramer =.532, p.000 - QSPL).

\section{Número de acções de formação profissional}

Relativamente ao número de acções de formação profissional per capita para quadros, nos últimos 3 anos, as empresas realizaram maioritariamente entre 2 a 5 acções de formação (QSPT - 59,4\%; QSPL - 61,9\% das empresas). De destacar a percentagem de empresas que realizaram mais de 5 acções de formação (QSPT - 25,0\%; QSPL - 23,8\%).

Considerando a formação por tipo de estratégia, o teste de Kruskal Wallis revela diferenças estatisticamente significativas $\left(X^{2}=8,167,2 \mathrm{gl}, \mathrm{p}<.05\right)$ entre as empresas que adoptaram predominantemente uma das estratégias sob estudo, para os QSPL. As empresas com estratégia de inovação são as que mais apostam na formação (número de acções), seguidas das que adoptam uma estratégia de qualidade, ambas com resposta modal na categoria 2 a 5 acções per capita. As empresas com estratégia de baixo custo têm o menor investimento em acções de formação, com moda situada em 1 acção de formação (Gráfico 1).

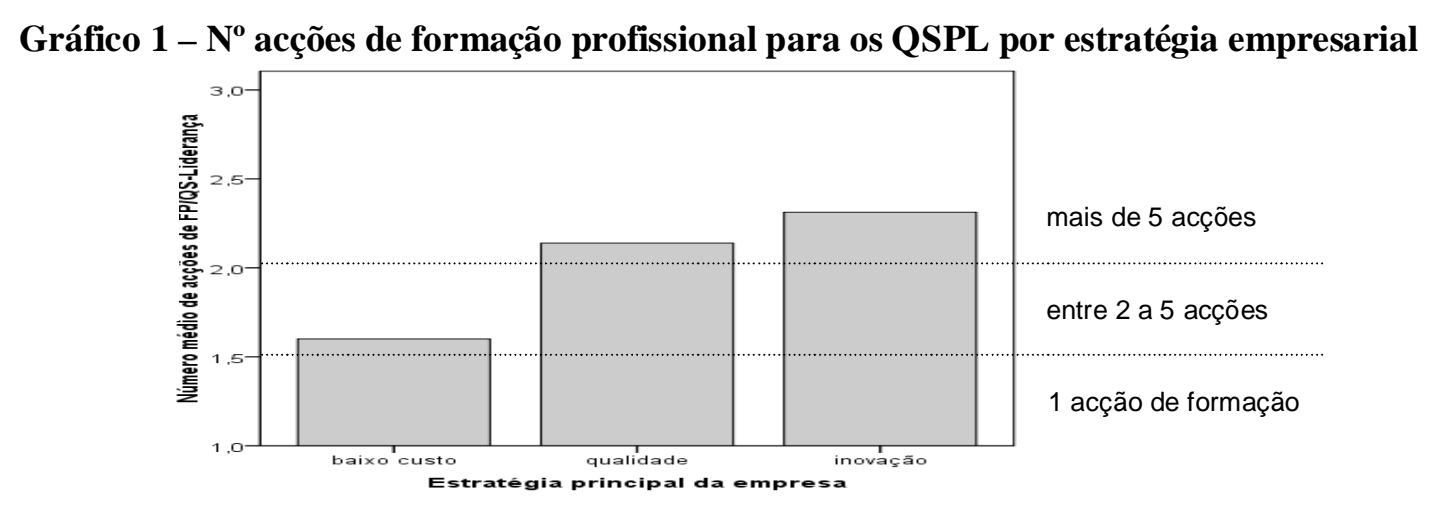

\section{Número de horas de formação profissional}

Procurámos ainda identificar qual o número de horas de formação profissional, per capita, para os QSPT e QSPL, nos últimos 3 anos. As empresas realizaram maioritariamente entre 50 a 99 horas de formação profissional (QSPT - 40,6\%; QSPL - 47,6\%). 
23 e 24 de Setembro de 2010

O teste de Kruskal Wallis $\left(X^{2}=7,653,2 \mathrm{gl}, \mathrm{p}<.05\right)$ revelou existirem diferenças estatisticamente significativas entre as empresas que adoptaram predominantemente uma das estratégias sob estudo, para os QSPL. Os resultados indicam que as empresas com estratégia de inovação apostam mais na formação (em número de horas), seguidas das que adoptam estratégia de qualidade, ambas com resposta modal na categoria "100 a 200" horas de formação per capita. As empresas com estratégia de baixo custo apostam menos em formação, com moda situada no intervalo de "< 50 horas" e "50 a 99" horas de formação per capita (Gráfico 2).

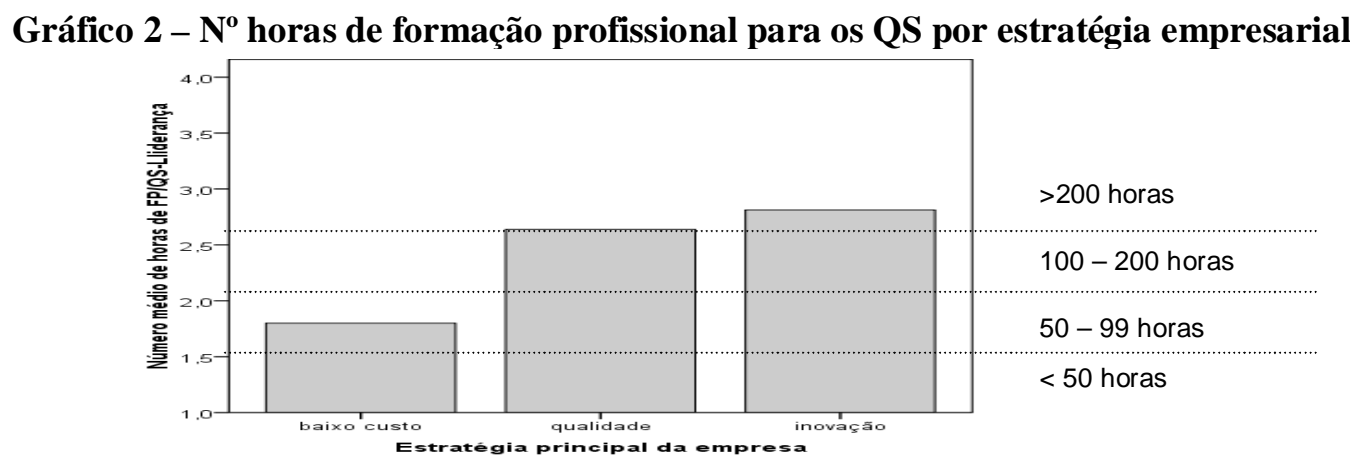

\section{Gestão de carreiras}

\section{Processos de desenvolvimento de carreiras}

Para a generalidade das empresas, os itens relacionados com a gestão de carreiras mais referidos, tomados como aqueles que se apresentam acima da média do grupo $(1,44)$, são a "avaliação da carreira pela empresa" (1,73), o "aperfeiçoamento da carreira através de programas de formação" $(1,54)$ e o "aperfeiçoamento da carreira através de preparação individual" $(1,47)$. Os restantes processos situam-se abaixo da média.

Considerando os processos de desenvolvimento de carreiras por estratégia empresarial, constatamos algumas diferenças. A "avaliação da carreira feita pelo trabalhador" é um processo preferencialmente aplicado às empresas que assentam a sua estratégia na inovação $(68,8 \%)$, em detrimento das empresas de baixo custo $(23,5 \%)$ e de qualidade $(38,5 \%)$. Quanto ao “aperfeiçoamento da carreira através da preparação individual" e ao "aperfeiçoamento da carreira através de programas de formação", a variação é segundo o binómio "empresas de 
qualidade e de inovação" versus "empresas de baixo custo", sendo a sua aplicação é muito reduzida nas empresas de baixo custo (76,5\% não aplica este processo).

Os testes realizados revelaram existir diferenças estatisticamente significativas entre as estratégias das empresas quanto à "avaliação da carreira feita pelo trabalhador" $\left(X^{2}=7,294,2 \mathrm{gl}\right.$, $\mathrm{p}<.05)$, ao "aperfeiçoamento da carreira através da preparação individual" " $\left(X^{2}=6,068,2 \mathrm{gl}\right.$, $\mathrm{p}<.05)$, e ao "aperfeiçoamento da carreira através de programas de formação" $\left(X^{2}=8,426,2 \mathrm{gl}\right.$, $\mathrm{p}<.05)$. Estes processos de gestão de carreiras variam e dependem da estratégia empresarial.

\section{Modalidades de progressão na carreira}

No inquérito aplicado foram identificados quatro tipos de modalidades de progressão na carreira dos QS. Foi realizada uma escala de Likert de 4 pontos cotada entre "nada importante" até "muito importante", sendo solicitado a sua categorização em termos de importância. Os itens são categorizados de forma independente, podendo co-ocorrer com o mesmo grau de importância.

Os resultados indicam que o item mais importante, tomado como aquele que se apresenta acima da média do grupo para todos os itens (2.6) é a "progressão por desempenho e mérito" (3.87). A menos valorizada é a "progressão por antiguidade" (1.94), indicando uma valorização do desempenho e do mérito na ascensão na carreira dos QS, o que está de acordo com o postulado pelas «PTED», em detrimento das tradicionais modalidades.

O teste de Kruskal Wallis revelou não existir diferenças estatisticamente significativas entre a progressão dos QS e a estratégia empresarial (Kruskal Wallis, p>.05 para todos os itens).

\section{Gestão das remunerações}

\section{Remuneração base}

Procurámos identificar o nível de remuneração base líquida para os quadros, distinguindo entre QSFT e QSFL. A remuneração base para os QS em função da estratégia empresarial adoptada, apresenta diferenças significativas entre as empresas (Teste V de Cramer =.536, p=.000; e Teste $V$ de Cramer $=.565, \mathrm{p}=.000$, para nível de remuneração base para QSPT e QSPL, 
respectivamente). A remuneração base dos QS cresce à medida que se caminha para as empresas com estratégia de qualidade e inovação.

São sobretudo as empresas com estratégia de inovação que tendem a pagar salários base superiores (de "2000 a 2499 euros" e de "2500 a 2999 euros"), quer se trate de QSPT quer QSPL. No pólo oposto estão as empresas com estratégia de baixo custo, as quais tendem a pagar salários base menos elevados, situando, preferencialmente, os salários nos intervalos de "1000 a 1499 euros" e "1500 a 1999 euros". No pólo intermédio estão as empresas com estratégia de qualidade, que privilegiam o pagamento de salários base na ordem dos "2000 a 2499 euros".

\section{Composição da remuneração}

Os resultados indicam que a composição da remuneração dos QS segue um padrão uniforme para os dois perfis de QS. Os itens mais importantes, tomados como aquelas que se apresentam acima da média do grupo (1.35 para QSPT; 1.37 para QSPL) são "seguros vários", “carro/combustível”, "prémios variáveis", "apoios financeiros à formação”.

Analisando as componentes da remuneração base para os QS em função da estratégia empresarial adoptada, existem diferenças estatisticamente significativas (Tabela 4). São sobretudo as empresas com estratégia de qualidade e inovação que tendem a utilizar os "prémios variáveis", "carro/combustível" e "apoios financeiros à formação", em detrimento das empresas que assentam a sua estratégia no baixo custo. Ao nível do "subsídio de desempenho" e dos "stock-options", apesar de pouco aplicados pelas empresas, são sobretudo aplicados pelas empresas com estratégia de inovação (56,2\% subsídio de desempenho, 31,2\% stock-options).

Tabela 4 - Testes do qui-quadrado e $\mathrm{V}$ de cramer

\begin{tabular}{|c|c|c|c|c|c|c|}
\hline & \multicolumn{3}{|c|}{ QS com perfil técnico } & \multicolumn{3}{|c|}{ QS com perfil liderança } \\
\hline & Chi-Square/ V Cramer & Gl & Sig. & Chi-Square/ V Cramer & $\mathrm{gl}$ & Sig. \\
\hline Prémios fixos &, $172(b)$ & 2 & 347 & ,161 (b) & 2 & ,393 \\
\hline Prémios variáveis & 7,938 (a) & 2 &, 019 & 8,829 (a) & 2 &, 012 \\
\hline Prémios por antiguidade &, 137 (b) & 2 &, 508 &, $137(b)$ & 2 &, 508 \\
\hline Subsídio de desempenho & 6,197 (a) & 2 & ,045 & 6,757 (a) & 2 &, 034 \\
\hline Stock-options &, $357(b)$ & 2 &, 010 & ,357 (b) & 2 & ,010 \\
\hline Participação nos resultados &, $220(b)$ & 2 &, 176 &, 066 (b) & 2 & 855 \\
\hline Carro/combustível & $12,023(a)$ & 2 & ,002 &, 475 (b) & 2 &, 000 \\
\hline Cartões de crédito &, 246 (b) & 2 &, 113 & 4,283 (a) & 2 & 117 \\
\hline Apoios financeiros à formação & 8,151 (a) & 2 & 0,17 & 7,697 (a) & 2 & 0,21 \\
\hline Seguros vários &, $118(b)$ & 2 & ,607 & ,058 (b) & 2 & ,886 \\
\hline
\end{tabular}

a) 0 cells $(, 0 \%)$ have expected count less than $5 . \mathrm{b}) \mathrm{V}$ de Cramer. 


\section{Formas de aumento salarial}

23 e 24 de Setembro de 2010

A forma de aumento salarial mais é utilizada pelas empresas é a sugerida pelas «PTED», ou seja, o "aumento individualizado segundo o mérito/desempenho" (66,2\% das empresas), seguida do "aumento geral segundo os resultados" (45,1\% das empresas). As formas menos praticadas são os "aumentos individualizados segundo conhecimentos/formação" e os "aumentos diferenciados segundo os resultados" (só preconizados por 2,8\% e 11,3\% das empresas, respectivamente).

As diferenças são evidentes se compararmos as formas de aumento salarial face à estratégia empresarial. O "aumento geral segundo as convenções" é aplicado sobretudo nas empresas com estratégia de baixo custo $(88,2 \%)$. O "aumento individualizado segundo o mérito/desempenho" é opção, sobretudo, nas empresas que assentam a sua estratégia na qualidade e na inovação (76,3\% e $87,5 \%$ respectivamente) dando, assim, dão seguimento ao definido pelas «PTED».

Há claramente uma associação entre o tipo de estratégia e as formas de aumento salarial de acordo com as "convenções colectivas" $\left(\mathrm{X}^{2}=24.820,2 \mathrm{gl}, \mathrm{p}=.000\right)$ e o "mérito/desempenho" $\left(\mathrm{X}^{2}=18.815,2 \mathrm{gl}, \mathrm{p}=.000\right)$. Relativamente às restantes formas de aumento salarial não existe tal associação, seguindo o mesmo padrão independentemente da estratégia adoptada.

\section{Conclusão}

O objectivo geral do estudo foi analisar a relação entre «PTED» dos QS e «estratégias empresariais» no sector de componentes para automóvel. Para tal realizámos uma revisão da literatura e uma pesquisa empírica. A revisão da literatura permitiu identificar o referencial teórico que sustenta a investigação, através da explicitação dos quadros teóricos e dos principais conceitos. A pesquisa empírica realizada permitiu retirar as seguintes conclusões:

- As empresas possuem diferentes estratégias principais para fazerem face ao mercado (estratégia de inovação, de qualidade e de baixos custos).

- Os QS são considerados agentes estratégicos de desenvolvimento das empresas.

- As «PTED», ao nível dos QS, caracterizam-se por: participação e acesso à informação; segurança no emprego (efectividade no tipo de contrato, com poucas saídas de QS); forte aposta na formação profissional; progressão na carreira contingente ao desempenho e remuneração contingente ao desempenho e ao mérito. 
- As «PTED», ao nível dos QS, diferem e são influenciadas pelas estratégias. As práticas de gestão dos QS são tendencialmente mais qualificantes nas empresas com estratégia de qualidade e inovação, por comparação com as empresas com estratégia de baixo custo.

Em suma, a articulação entre «estratégias empresariais» e «PTED» é consequência do próprio processo de formulação e implementação estratégica, bem como do papel dos QS enquanto agentes estratégicos de desenvolvimento das empresas. Ao possuírem diferentes estratégias, as empresas revelaram ainda possuir diferentes formas de as preconizar. Estratégias empresariais e «PTED» sustentam e são sustentadas, por e entre si, num processo de retroalimentação mútua.

\section{Bibliografia}

Becker, B. E., Huselid, M. A., Ulrich, D. (2001). Gestão de Pessoas com Scorecard: interligando pessoas, estratégia e performance. Edição Campus.

Becker, B. E., Huselid, M. A. (1999). An overview: strategic human resource management in five leading firms. Human Resource Management, 38 (4), pp. 287-301.

Bilhim, J. A. (2004). Gestão estratégica de recursos humanos. Universidade Técnica de Lisboa, Lisboa: ISCSP.

Ciavarella, M. (2003). The adoption of high-involvement practices and processes in emergent and developing firms: a descriptive and prescriptive approach. Human Resources Management, 42 (4), pp. 337-356.

Cordeiro, J. P. (2008). Gestão de recursos humanos e estratégias empresariais: os quadros superiores das empresas do sector de componentes para automóvel. Tese de Doutoramento em Sociologia, Lisboa: ISCTE.

Delery, E., Shaw, J. (2001). The strategic management of people in work organizations: review synthesis, and extension. Research in personnel and Human Resources Management, 20, pp. 165-197.

Huang, T. C. (2002). The effects of linkage between business and human resource management strategies. Personnel Review, 30 (2), pp. 132-151.

Husellid, M. A., Jackson, S. E., Schuler, R. S. (1997). Technical and strategic human resource management effectiveness as determinants of firm performance. Academy of Management Journal, 40 (1), pp.171-188.

Maroco, J. (2007). Análise estatística com utilização do SPSS. Edições Sílabo, $3^{\text {a }}$ edição.

Morais, F. (1998). Evolução dos modelos de gestão. Lopes, H. (coord.), As modalidades de empresa que aprende e empresa qualificante. Estudos e Análises, no 12, cap. 9, Lisboa: OEFP.

Pernin, D. (1985). La gestion des cadres - acteurs de leur carrière. Paris: Les Éditions d'Organisation.

Pil, F., MacDuffie, J. P. (1996). The adoption of High-Involvement work practices. Industrial Relations, 35 (3), pp. 423-456.

Porter, M. (1980). Competitive strategy: techniques for analysing industries and competitors. New York: Free Press.

Porter, M. (1985). The competitive advantage. Free Press, New York.

Schuler, R., Jackson, S. (1987). Linking competitive strategies with human resource practices. The Academy of Management Executive, 1 (3), pp. 207-219.

Thompson, Jr, Strickland III, A. (2000). Planejamento estratégico: elaboração, implementação e execução. São Paulo: Pioneira.

Torrington, D. (2002). Strategic human resource management. Torrington, D., Hall, L., Taylor, S., Human Resource Management, cap. 3, Great-Britain: Prentice Hall, Fifth Edition, pp. 30-47.

Wright, P., McMahan, G. (1992). Theoretical perspectives for strategic human resource management. Journal of Management, 18 (2), pp. 295-320. 